understanding the full burden of disease among them has been challenging as direct estimates of Men who Have Sex with Men (MSM) numbers in the general population have been largely unavailable. We describe the population of Men who Have Sex with Men (MSM) in New York City, compare their demographics, risk behaviours, and new HIV and primary and secondary (P\&S) syphilis rates with those of men who have sex with women (MSW), and examine trends in disease rates among Men who Have Sex with Men (MSM).

Methods Population denominators and demographic and behavioural data were obtained from population-based behavioural surveys during 2005-2008. Numbers of new HIV and P\&S syphilis diagnoses were extracted from citywide disease surveillance registries. We calculated overall, age- and race/ethnicity-specific case rates and rate ratios for Men who Have Sex with Men (MSM) and MSW, and analysed trends in Men who Have Sex with Men (MSM) rates by age and race/ethnicity.

Results The average prevalence of same-sex behaviour among sexually active men during 2005-2008 (5.0\%; 95\% CI 4.5 to 5.6) differed by age (peaking at $8 \%$ among 40-49-year-old men) and race/ethnicity (2.3\% among non-Hispanic black men; $7.4 \%$ among non-Hispanic white men). Compared to MSW, Men who Have Sex with Men (MSM) differed significantly on all demographics and reported a higher prevalence of condom use at last sex and of HIV testing, but also more sex partners; $38.4 \%$ of Men who Have Sex with Men (MSM) and $13.6 \%$ of MSW reported $=3$ partners in the last year $(p<0.001)$. Men who Have Sex with Men (MSM) HIV and P\&S syphilis rates were 2526.9/100 000 and 707.0/100 000, each of which was over 140 times MSW rates. Rates were highest among young and black Men who Have Sex with Men (MSM) (See Abstract LBO-1.5 table 1). Over 4 years, HIV rates more than doubled and $\mathrm{P} \& \mathrm{~S}$ syphilis rates increased sixfold among 18-29-yearold Men who Have Sex with Men (MSM) to reach 8870.0/100 000 and 2900.4/100 000 in 2008, respectively.

Conclusions The substantial population of Men who Have Sex with Men (MSM) in NYC is at high risk for transmission of sexually transmitted infections given high disease rates and ongoing risk behaviours. There is significant overlap between HIV and $\mathrm{P} \& \mathrm{~S}$ syphilis epidemics in NYC with the relatively small subgroups of young and non-Hispanic black Men who Have Sex with Men (MSM) disproportionately affected. Integration of HIV and STD case data would allow for better identification and characterisation of the population affected by these synergistic epidemics. Intensified and innovative efforts to implement and evaluate prevention programs are required.

\section{LB0-1.6 EFFICACY OF THE QUADRIVALENT HPV VACCINE TO PREVENT ANAL INTRAEPITHELIAL NEOPLASIA AMONG YOUNG MEN WHO HAVE SEX WITH MEN}

doi:10.1136/sextrans-2011-050119.6

\section{S Goldstone. Albert Einstein College of Medicine, New York, USA}

Introduction Most anal cancers are associated with HPV, particularly HPV 16. The incidence of anal cancer is increased among men who have sex with men (MSM) compared with the general population. Screening and treatment of anal intraepithelial neoplasia (AIN), the anal cancer precursor, are not yet standard of care, and prevention efforts are needed to reduce the incidence of anal cancer. We tested the quadrivalent HPV vaccine to determine its ability to reduce the incidence of AIN/anal cancer.

Methods 598 MSM aged 16-26 years with five or fewer lifetime sex partners were randomised to receive vaccine or placebo at enrolment, month 2 and month 6. Subjects underwent detailed anogenital exams and HPV sampling from the penis, scrotum, perineal/perianal and anal canal at enrolment, month 7 and at 6-month intervals afterwards. Efficacy analyses were performed in a per-protocol (PPE) population (sero-negative and DNA-negative from day 1 through month 7 to the relevant vaccine HPV type) and in all enrolees in an intent-to treat (ITT) analysis. Median follow-up of the PPE population was 2.5 years (post-dose 3 )

Results Vaccine efficacy against HPV 6/11/16/18-related AIN and anal cancer in the PPE population was $77.5 \%$ (95\% CI 39.6 to 93.3) (5 vaccine cases vs 24 placebo cases). Efficacy against high-grade AIN (AIN $2+$ ) was $74.9 \%$ (95\% CI 8.8 to 95.4$)$. In the ITT population the efficacy was $50.3 \%$ (95\% CI 25.7 to 67.2 ) and $54.2 \%$ (95\% CI 18.0 to 75.3), respectively. No anal cancer was seen in either treatment group. Conclusions These results demonstrate that the quadrivalent HPV vaccine is efficacious in preventing AIN related to HPV 6/11/16/18 in MSM subjects naïve to vaccine HPV types at enrolment, as well as in an ITT population. The quadrivalent HPV vaccine may be a useful measure to reduce the incidence of anal disease in at-risk populations.

\section{Late breaker poster session}

\section{LBP-1.01 THE INTERDICTION PROJECT: AN INNOVATIVE PROGRAM FOR HIV + PERSONS TO REDUCE HIV AND STD CO-INFECTION AND IMPROVE HIV TREATMENT OUTCOMES PHASE II}

doi:10.1136/sextrans-2011-050119.7

S Frimpong, M Wilson, L M Mitchell-Samon, T Azarian. Duval County Health Department

Background/Objectives STD/HIV field investigations and the initial findings of Interdiction Project reveal that some persons with HIV infection have unprotected sex, multiple anonymous sex partners, and don't disclose their HIV status. Consistent condom use is low; recurrent co-infection with STDs and HIV transmission to sex partners is high. The Interdiction Project is a clinic-based, individuallevel HIV intervention that combines linkage for treatment adherence/risk reduction education and testing with ongoing monitoring of patient care and epidemiologic data system. This project targets HIV positive persons who present with a new STD or history of unprotected sex

Methods This is a prospective STD intervention follow-up study of 69 patients referred by HIV/AIDS and STD clinic providers and STD/HIV investigation specialist. Project staff conducted initial HIV/AIDS and STD transmission prevention and treatment monitoring knowledge plus risk assessment, intensive practical STD and HIV/AIDS health education, condom negotiation, condom skill training, sex partner elicitation for rapid HIV testing and linkage to care. STD and STD morbidity rates 18 months prior to follow-up were compared to 18 months of follow-up after referral. We analysed follow-up data to determine the STD rates, undetectable HIV viral loads and how Interdiction Project may help reduce the spread of HIV and STD co-infections.

Results Initial findings revealed high STD morbidity since HIV diagnosis and 26 clients had 48 STDs before referral. Overall STD rate was $50.7 \%(35 / 69)$ at referral, 14\% consistent condom use always but $96 \%$ intend to use condoms and sex partner HIV positivity rate was 30\% (6/20). HIV genotype resistance was 34\% (14/ 41). At 18 months of follow-up, significant overall STD morbidity rate reduced from $74.3 \%$ to $25.4 \%$, RR 0.34 (95\% CI 0.22 to 0.52 ) and RR of STD reduced from $72.5 \%$ to $23.2 \%$, RR 0.32 (95\% CI 0.20 to 0.50$)$. Achieving higher perfect knowledge score from pre- to posteducation knowledge assessment correlated with reduction in STD and STD morbidity. Significant STD rates reduction was noted for many risk categories. Clients who had undetectable viral load on HAART increased from $50 \%(17 / 34)$ at referral to $88.2 \%(30 / 34)$ at 6-9 months and $77.4 \%$ (24/31) from 13 to 18 months of follow-up. 
Conclusion/Implication Interdiction Project applies concepts of clinical epidemiology similar to in-patient hospital infection control processes and may help HIV/AIDS patients to achieve undetectable HIV viral load, reduce STD co-infection and HIV transmission. The findings suggest that epidemiologic work to reduce the spread of HIV, STD co-infection and resistant HIV must be an important component of HIV/AIDS programs and will require collaborative work by medical providers and intensive practical health education by knowledgeable HIV/AIDS health educator/epidemiologist.

\section{LBP-1.02 ACCEPTABILITY AND APPEAL OF INTERNET-BASED STI AND HIV TESTING AMONG A SAMPLE OF GAY MEN AND CLIENTS ATTENDING STI CLINICS IN BRITISH COLUMBIA}

doi:10.1136/sextrans-2011-050119.8

${ }^{1} \mathrm{~T}$ S Hottes, ${ }^{1} \mathrm{M}$ Gilbert, ${ }^{1} \mathrm{M}$ Bondyra, ${ }^{1} \mathrm{D}$ Haag, ${ }^{2} \mathrm{~J}$ Shoveller. ${ }^{1} B C$ Centre for Disease Control, Vancouver, Canada; ${ }^{2}$ University of British Columbia, Canada

Background Several jurisdictions globally have implemented internet-based STI and HIV testing with good uptake and reach into untested populations. In British Columbia, a similar program is under development to offer online access to chlamydia, gonorrhoea, syphilis, and HIV testing. This program aims to increase the frequency of testing in individuals at risk of infection and ease demand on clinic-based services by providing an accessible option for screening asymptomatic persons. We conducted a series of focus groups in spring 2011 to determine the acceptability of various aspects of the internet testing model and of the service overall. Methods Two types of prospective internet testing clients living in the Vancouver area were recruited for focus groups: self-identified gay/bisexual/two-spirit men (hereafter "gay men") and STI clinic clients. Participants were presented with a brief description of the internet testing model, after which a structured interview guide was used to address the following domains: willingness to provide personal information online, ways to engender trust in the service, comfort with different ways of delivering results, interest in specific features, appeal of the service, and willingness to use the service.

Results Most of the 31 participants to-date (ages $21-70$ years) were gay men (81\%) and reported post-secondary education (74\%). All were self-described active internet users and had been tested previously for HIV or other STI. Overall, the concept of internet testing was thought to be acceptable and most participants expressed interest in using the service when it becomes available. Participants indicated that internet testing would be convenient and could enhance the accessibility of STI testing, particularly for people living outside of urban centres. Some noted that an internet approach would also have the potential to allay embarrassment that can arise when seeking STI testing in a face-to-face clinical setting. There was strong interest in assuring anonymity for those using the proposed internet testing service. Participants also emphasised the importance of providing information related to HIV diagnosis and referrals to peer support and counselling for those waiting for and receiving test results.

Conclusions Internet testing was deemed to be acceptable and desirable and appears to hold promise as a means to complement to existing clinic-based STI testing services in Vancouver, Canada.

\section{LBP-1.03 "NO ONE WAS THERE TO CARE FOR US" ASHRAYA'S RESPONSE TO ITS MEMBERS' NEEDS IN TERMS OF CARE AND SUPPORT}

doi:10.1136/sextrans-2011-050119.9

C Chevrier. Université de Montréal, Montréal, Canada

Background Sex workers (SWs) have been identified as a "high-risk group" and considerable effort has been invested in promoting "safer sex" among this group. However, their specific concerns and identities vanish in broader public health policy discussions related to treatment and particularly care. Ashodaya Samithi, a SWs organisation based in Mysore, Karnataka, has been providing extensive care and support (C\&S) services to SWs and "non-SWs" living with HIV through their sub-wing organisation, Ashraya, since 2008. Considering the enduring stigma attached to SWs, what motivates individuals to seek health services from Ashraya?

Methods 40 in-depth interviews (including 10 with non-SWs) were conducted with Ashraya members in 2011 to explore how Ashraya responds to the needs of its members in terms of C\&S. Content analysis of the transcripts was completed to sort out themes.

Results Analysis shows that in spite of scarce resources, the accessibility, effective outreach efforts, and commitment and reliability of Ashraya's volunteers are major components of its growing popularity.

Conclusions In the near-absence of non-discriminatory, affordable C\&S for people living with HIV/AIDS, Ashraya offers much needed relief to its members. The potential role of SWs community-based organisations in providing $C \& S$, which goes beyond safer sex promotion, needs to be accounted for within national-level policy planning and in evaluating existing programs.

\section{LBP-1.04 INTERNATIONAL VALIDATION OF AMPLISENS NAAT SYSTEMS MANUFACTURED IN RUSSIA FOR DIAGNOSIS OF NEISSERIA GONORRHOEAE, CHLAMYDIA TRACHOMATIS, MYCOPLASMA GENITALIUM AND TRICHOMONAS VAGINALIS}

doi:10.1136/sextrans-2011-050119.10

${ }^{1} \mathrm{M}$ Domeika, ${ }^{2} \mathrm{~A}$ Savicheva, ${ }^{2} \mathrm{E}$ Shipitsyna, ${ }^{3} \mathrm{C}$ Y Chen, ${ }^{4} \mathrm{~J}$ Skov-Jenssen, ${ }^{5} \mathrm{O}$ Hjelmevoll, ${ }^{4} \mathrm{R}$ Ballard, ${ }^{6} \mathrm{M}$ Unemo. ${ }^{1}$ Uppsala University, Uppsala, Sweden; ${ }^{2}$ Institute of Obstetrics and Gynecology DI Ott, St Petersburg, Russian Federation; ${ }^{3}$ Centers for Disease Control and Prevention, Atlanta, Georgia, USA; ${ }^{4}$ State Serum Institute, Copenhagen, Denmark; ${ }^{5}$ University Hospital of North Norway, Tromso, Norway; ${ }^{6}$ Örebro University Hospital, Örebro, Sweden

Objectives Data regarding the performance characteristics of STI diagnostic tests used in Eastern Europe are very scarce. The objective of this study was to validate the AmpliSens test systems (Central Research Institute for Epidemiology, Moscow, Russia), compared to internationally acknowledged and validated tests, for genetic detection of $N$ gonorrhoeae (NG), C trachomatis (CT), $M$ genitalium (MG) and $T$ vaginalis (TV).

Methods In total, 319 females and 127 males were recruited. For evaluation of the TV tests, additional 33 patients with diagnosed trichomoniasis at dermatovenereological dispensaries (VD) were involved. Conventional AmpliSens PCR assay (cPCR), real-time PCR assay (rtPCR), and a real-time nucleic acid sequence-based amplification assay for detection of NG or CT were evaluated. Furthermore, AmpliSens cPCR and rtPCR for detection of MG or TV were tested. As international reference tests an internationally validated real-time $N$ gonorrhoeae porA pseudogene PCR, Cobas Amplicor CT PCR and LightMix 480HT CT PCR, MG rtPCR targeting the $\mathrm{MgPa}$ adhesin gene with positive results confirmed by two MG cPCRs targeting the $16 \mathrm{~S}$ rRNA gene and MgPa gene, and a TV rtPCR targeting a TV specific repeat DNA fragment, respectively, were used.

Results The overall prevalence of CT infection was $12.6 \%$. The AmpliSens CT NAATs and the reference methods displayed a high level of concordance $(97.9 \%-99.2 \%)$. The prevalence of NG was $2.7 \%$ and $16 \%$ among the urogenital and extragenital samples, respectively. The AmpliSens NG NAATs and the reference method 